\title{
A Rare Disease Patient Manager
}

\author{
Pedro Lopes ${ }^{1}$, Rafael Mendonça ${ }^{1}$, Hugo Rocha ${ }^{2}$, Jorge Oliveira ${ }^{2}$, Laura \\ Vilarinho $^{2}$, Rosário Santos ${ }^{2}$, José Luís Oliveira ${ }^{1}$
}

\author{
${ }^{1}$ DETI/IEETA, Universidade de Aveiro, \\ 3880-193 Aveiro, Portugal \\ \{pedrolopes,rmendonca, jlo\}@ua.pt \\ ${ }^{2}$ Centro de Genética Médica Jacinto Magalhães, INSA, \\ 4099-028 Porto, Portugal \\ \{hugo.rocha, jorge.oliveira, laura.vilarinho, rosario.santos\}@insa.min-saude.pt
}

\begin{abstract}
The personal health implications behind rare diseases are seldom considered in widespread medical care. The low incidence rate and complex treatment process makes rare disease research an underrated field in the life sciences. However, it is in these particular conditions that the strongest relations between genotypes and phenotypes are identified. The rare disease patient manager, detailed in this manuscript, presents an innovative perspective for a patient-centric portal integrating genetic and medical data. With this strategy, patient's digital records are transparently integrated and connected to wet-lab genetics research in a seamless working environment. The resulting knowledge base offers multiple data views, geared towards medical staff, with patient treatment and monitoring data; genetics researchers, through a custom locus-specific database; and patients, who for once play an active role in their treatment and rare diseases research.
\end{abstract}

Keywords: Rare Diseases, Genetic Mutations, LSDB, Personalized Medicine.

\section{Introduction}

Biomedical research states that a rare disease is a particular condition affecting at most 1 in 2000 patients. The European Organization for Rare Diseases (EURORDIS) estimates that there are approximately 6000 to 8000 rare diseases, affecting about $6 \%$ to $8 \%$ of the population. Within these, about $80 \%$ are caused by genetic disorders. Due to the reduced incidence of each individual disease, it is difficult for patients to find support, both at clinical and psychological level [1]. The existence of a small number of patients for each rare disease also delays the creation of adequate research studies as it is difficult to identify and coordinate a correct cohort $[2,3]$. Despite the low statistic impact regarding these diseases, the 
combined amount of patients suffering from one of these rare diseases is considerably high.

The Portuguese National Programme for Rare Disorders, approved in 2008, strives towards a concerted system for rare disease tracking and patient monitoring. This endeavor relies heavily on a platform designed to gather information from the different stakeholders involved in rare disease patient care, ranging from diagnostic labs to health providers up to policy-makers. The set of requirements behind such a large-scale project touches miscellaneous research areas. The combination of heterogeneous data from medical reports with patient records and genetic datasets triggers problems in the areas of privacy and security, service composition and data integration.

The solution we envisage relies on edge-of-breed software engineering strategies to provide a seamless working environment for everyone involved in rare disease research. The platform, a rare disease patient manager, collects and connects data for patients during their entire stay in the system. This widespread information collection, including medical reports with the initial diagnosis, patient genetic test results or the patient treatment program, is fused into a single knowledge base. From there, distinct data views are provided to distinct user roles, each accessing its own private and independent web information system.

This Portuguese case study revolves around the creation of reference centres. These are more than a single physical institution, enabling the cooperation and networking amongst several public entities in the Portuguese health system and detaining a wealth of data on patients diagnosed with known rare diseases. This organizational structure, involving efforts from multidisciplinary teams, permits the best procedures to be available, simplifying processes and minimizing the resources needed. With the innovative rare disease patient manager proposed in this manuscript, the reference centres will be endowed with the required set of software tools to study the underlying rare disease causes and adverse effects and, above all, vastly improve patient care.

\section{Overview}

The importance of a patient's genetic records in diagnosis and resolution of rare diseases has taken medicine to a level where wet-lab research is crucial to unravel disease causes and consequences. Hence, databases emerging with information about human genome, such as the HGMD (Human Gene Mutation Database) [4] or the 1000 Genomes Project [5] retain growing relevance. It is important to collect their public data in novel biomedical software, enabling its usage on the daily medical workflow. Despite this growing, the absence of public medical reports, specially regarding rare disease patients, indicates the vulnerability of these patient groups [6]. 
Personalized medicine requires the combination of data from both these fields along with individual patient information. Data from sequence mutations, genes and drug interactions - the genotype - must be combined with data from electronic health records (EHR), DICOM imaging and disease-specific patient data - the phenotype. Henceforth, the patient-centric rare disease patient manager offers a unique holistic view promoting the collaboration of multiple entities towards the study of rare diseases and assessment of patients' evolution - Fig. 1.

The de facto standard in rare disease software is Orphanet [7], a web platform directed to the general public, health professionals and patients, to inform about orphan drugs and rare diseases. It also displays information on specialized consultations, diagnostics, research projects, clinical trials and support groups. Diseasecard [8] is another portal aggregating information regarding rare diseases and pointing to key elements for both the education and the biomedical research field.

Along with these applications, we must take in account EHRs, gathered from miscellaneous health information systems, containing clinically relevant patient information. Only by mining these reports and collecting them for future analysis we will be able to provide the adequate knowledge set for clinicians involved in the reference center.

Locus-specific databases (LSDB) are gene-centric applications, focused on providing variant-related features, such as store and search, to gene curators. They are usually used by a group of researchers in collaboration, with expertise in a particular gene or genotype, and provide a valuable tool for analysis of gene expression and phenotype, both in normal conditions and disease [9].

Concomitant with the systematic, widespread collection of patient data, LSDBs

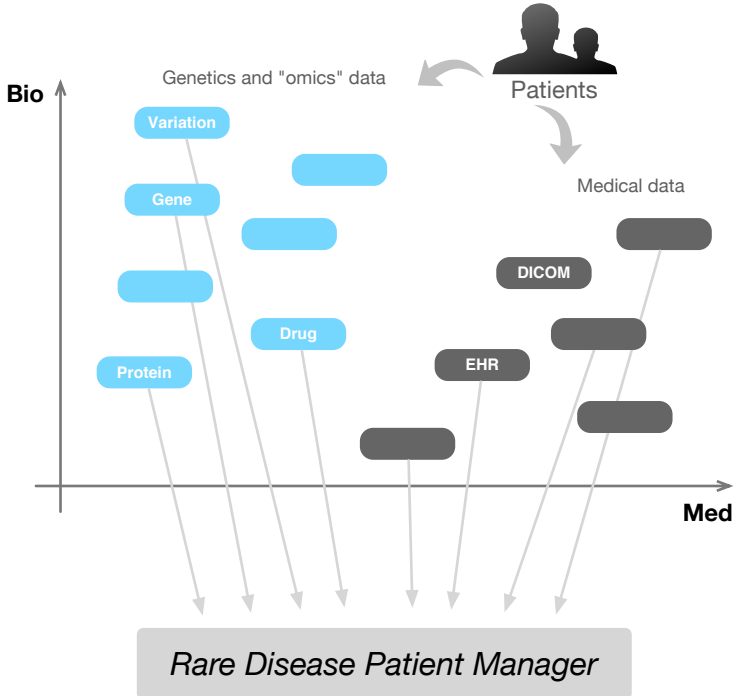

Fig. 1. The rare disease patient manager seamlessly integrates a collection of patient-centric data, combining the quadrants of genetics and "omics" information with miscellaneous medical data in a centralized knowledge base. 
ultimate goal is a contribution to the global effort of the Human Variome Project (HVP), which aims to collect, curate and distribute all human genetic variation affecting health [10-12].

The goal behind the creation of reference centres is to connect individuals from disparate research areas and specialized in a particular rare disease. Hence, a reference centre is composed of multidisciplinary teams with access to resources for the diagnosis, treatment and medical monitoring of patients.

When a patient, whose clinical conditions signal the presence of a known rare disease, arrives for medical screening, he is directed to the reference centre, initiating his presence in the rare disease patient manager. Initial contact with the clinicians permits the analysis of symptoms and the evaluation of future treatment scenarios. Along with the clinical analysis, patients are sent for genetic testing labs, where their genetic sequence will be sampled and explored, searching for well-known mutation and other biomarkers. The treatment of rare diseases at this level requires a deep collaboration between clinicians and genetics researchers.

\section{Information Workflow}

The rare disease patient manager provides a unified set of tools ranging from the integration of patient genetic and clinical data to the various independent data access views - Fig. 2.

Rare disease information in the patient manager knowledge base is built from OMIM, Online Mendelian Inheritance in Man [13], and HGNC, HUGO Gene Nomenclature Committee [14]. To cope with the increasing data in this field, new tools have emerged, namely MUTbase [15], Universal Mutation Database (UMD) [16] and Leiden Open-source Variation Database (LOVD) [17]. LOVD innovates with the "LSDB-in-a-box" approach: it is offered as a downloadable software package containing the full set of tools required for the deployment of a local locus-specific database. Using LOVD within the rare disease patient manager enables the quick arrangement of an in-house LSDB, allowing the registration of genetic mutations, linking them to patients in the rare disease patient manager. The resulting knowledge network, with direct connections from mutations to patients up to diseases represents a true complete view over a rare disease influence area.

When dealing with rare disease patients, the anonymity and privacy of data are regarded as imperative. To cope with the caveat of aggregating all data in a single knowledge base, the patient manager provides distinct "data views" over acquired information.

Each view is assigned to a distinct user-role, and enables/disables access to certain features or data-types. For example, with this approach, reference centre coordinators are differentiated from diagnostic lab technicians: the former are able to manage patient monitoring forms whereas the latter can only access the system's LSDB. 


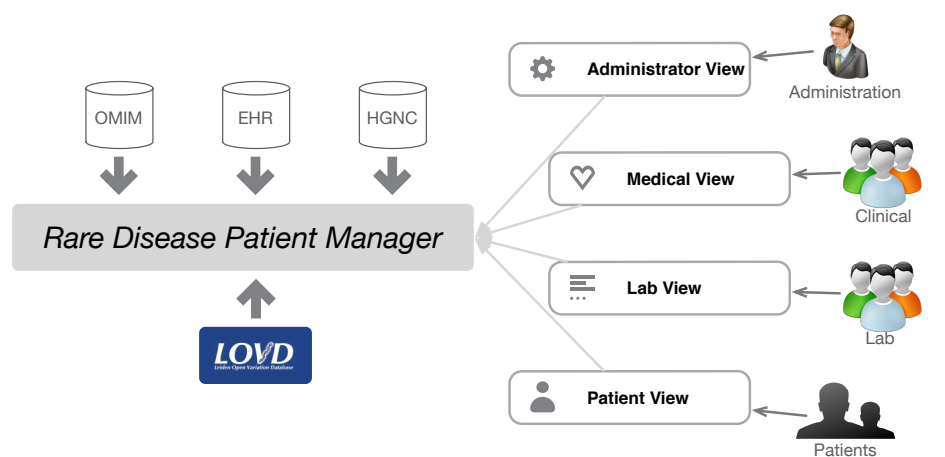

Fig. 2. Data aggregated in the rare disease patient manager is provided to various stakeholders, each assigned a distinct role and with its respective data views.

Furthermore, by placing the patient as the central element of this system, he is given the power to explore and control his data. Given the personal nature of collected data, patients have the option of not sharing their data to statistical analysis or, going the opposite way, and liberate their personal records to whomever they want within the reference centre team. Moreover, with the foreseen advances in personal electronic health records, patients will be able to get their data out of the system and share it with family, physicians or other information systems.

\section{Architecture}

The rare disease patient manager acts as a centralized, patient-centric, information portal. To reduce data distribution and increase the overall coherency, the system is deployed as a portal of websites. Leveraging on modern web information technologies, each reference centre is able to launch any number of rare diseaseoriented websites, each with its own customized rare disease patient management infrastructure. Despite being a part of the same physical architecture, each rare disease patient manager application is virtually independent and unaware of its sibling systems.

In parallel with the main rare disease patient manager system there is a LOVD server. This server enables the creation of new customized locus-specific databases that can be used independently. Hence, genetic lab teams within the reference centre can use their own LSDBs without interfering with the overall rare disease patient manager architecture.

Privacy and security are major requirements inherent to the rare disease patient manager model. Consequently, distinct types of users are given distinct roles. Role assignment is a responsibility of the reference centre coordinator. This role-based strategy allows for a transparent data access division: users assigned with a given role can only access features and data that are available to that role.

To complement the LSDB independent usage with the rare disease patient manager, the transition between systems needs to be transparent. For this matter, 
an innovative data sharing strategy enables cross authentication between the rare disease patient manager and the independent LOVD instances.

\section{Discussion}

The rare disease patient manager, overviewed in Fig. 3, provides a solid breakthrough in the seamless integration of genetic, medical and patient data. The patient registry, centralizing medical and genetic data in a single system, delivers a fresh and unique perspective to various types of researchers involved in the study of rare diseases. This provides comprehensive analysis and medical monitoring of patients from the moment they are registered in the system.

In addition to the aforementioned personalized genetic testing datasets, diseasespecific templates can be created to monitor a broad number of items. This permits the creation of custom forms at each rare disease patient manager, enabling both the tracking of individual rare disease markers, e.g. neuromuscular disorder level, or general phenotypic traits, e.g. blood type or pressure. Each tracking form contains the title, a short description and a set of fields. The fields can be of different types, mimicking the final user interaction: text, true/false, number, multiple selectable options or enumerations. With these fields, the system aims to respond to the overall needs of form personalization.

The innovative integration of gene-centric LSDBs with patient provides a tighter connection between a patient's genetic profile and his medical condition. With this, information collected in medical studies is complemented with genetic

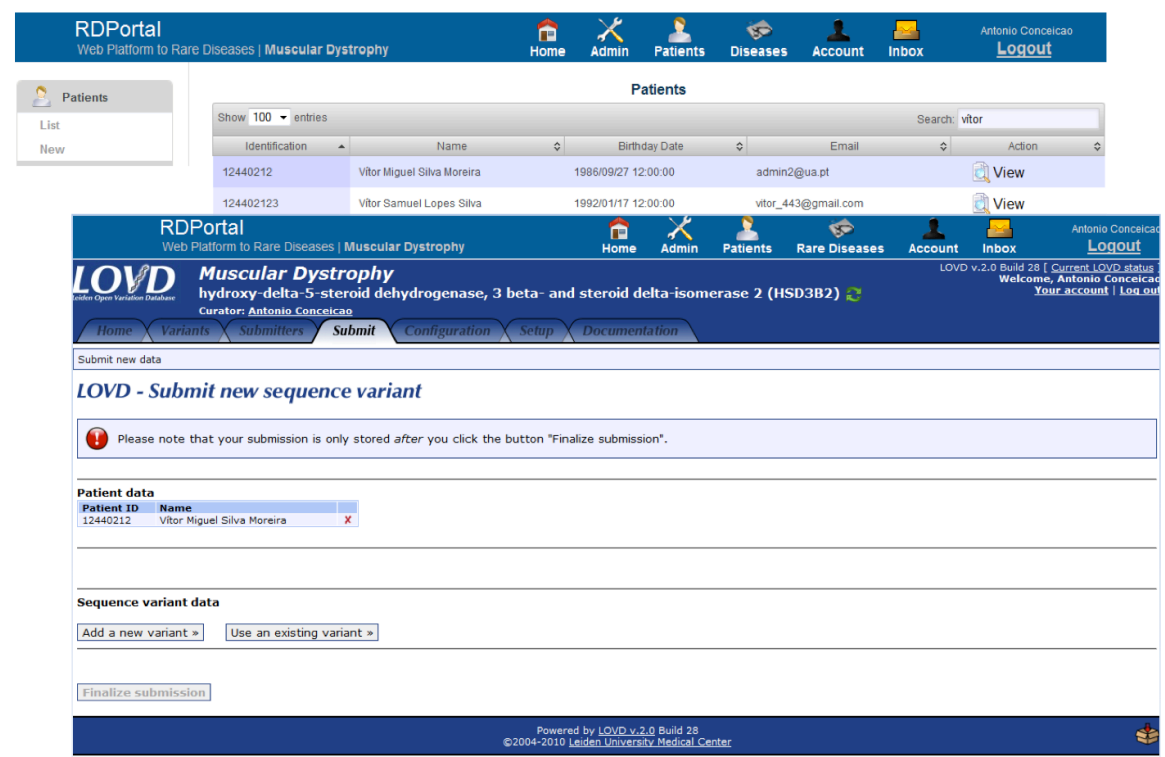

Fig. 3. Rare disease patient manager prototype interface highlighting the patient browser, on top, and the independent LOVD application, at the bottom. 
analysis carried out by wet-lab experts. As a result, this distinctive combination of data and custom disease tracking supports the creation of modern standardized rare disease monitoring procedures, aiding in the achievement of a more accurate diagnosis and of an improved patient care.

One obvious advantage of this system is its web connectivity, which enables the different participants to submit and update the clinical and mutational information in a central database using secure protocols. Simultaneously this information can be accessed and retrieved in real-time by its registered and authorized users. The general purpose solutions currently available are usually focused on intra-institutional use and do not incorporate such features. In addition, due to the diseases' specificities, the system design/architecture should be as flexible as possible. This platform is actually a "core-system" where the different reference centers can customize the structure according to their specific needs and workflow. Finally, it should be taken into account that genetic information management modules are not usually included in general laboratory or patient management systems. The mutational data integrated in this centralized platform can also be easily transferred to an international LSDB or central database.

\section{Conclusions}

Rare diseases are seldom qualified as mainstream life sciences research. Their specific features require novel research strategies involving the cooperation of researchers from the clinical, genetics and pharmaceutical domain. Despite successful advances on the last two decades, adequate information structure to support these multidisciplinary teams is severely absent. Information from diseases, symptoms, diagnostics, drugs and underlying genetic impact is missing or poorly available. The rare disease patient manager platform described in this manuscript aims to overcome these difficulties. This fusion of data from disparate research fields is of paramount importance to physicians, genetics researchers and, consequently, indispensable to patient care.

For clinicians, this system represents a breakthrough in rare disease patient data management. Clinicians can now track and manage patient data, treatments, diagnostics, procedures and genetic profiles in an integrated fashion. As for gene curators and wet-lab researchers, the system enables the usage of locus-specific databases in an independent yet complementary way, thus enriching patient's medical profiles. At last, for patients, being actively included in their disease-monitoring workflow, in the research for better treatments and with total control over personal data, represents a new standard in personalized medicine.

Acknowledgments The research leading to these results has received funding from the European Community's Seventh Framework Programme (FP7/2007-2013) under grant agreement no. 200754 - the GEN2PHEN project. 


\section{References}

1. Nabarette H, Oziel D, Urbero B, Maxime N, Aymé S (2006) Use of a directory of specialized services and guidance in the healthcare system: the example of the Orphanet database for rare diseases]. Revue d'épidémiologie et de santé publique 54 (1):41

2. Schieppati A, Henter JI, Daina E, Aperia A (2008) Why rare diseases are an important medical and social issue. The Lancet 371 (9629):2039-2041

3. Burke W (2002) Genetic testing. N Engl J Med 347 (23):1867-1875

4. Krawczak M, Ball EV, Fenton I, Stenson PD, Abeysinghe S, Thomas N, Cooper DN (2000) Human gene mutation database - a biomedical information and research resource. Human mutation 15 (1):45-51

5. Via M, Gignoux C, Burchard EG (2010) The 1000 Genomes Project: new opportunities for research and social challenges. Genome Medicine 2 (1):1-3

6. Schneeweiss S, Avorn J (2005) A review of uses of health care utilization databases for epidemiologic research on therapeutics. Journal of clinical epidemiology 58 (4):323-337

7. Seoane-Vazquez E, Rodriguez-Monguio R, Szeinbach SL, Visaria J (2008) Orphanet Journal of Rare Diseases. Orphanet journal of rare diseases 3:33

8. Oliveira JL, Dias GMS, Oliveira IFC, Rocha PDNSd, Hermosilla I, Vicente J, Spiteri I, Martin-Sánchez F, Pereira AMMdS DiseaseCard: A Web-based Tool for the Collaborative Integration of Genetic and Medical Information. In: 5th International Symposium, ISBMDA 2004: Biological and Medical Data Analysis, 2004. pp 409-417

9. Claustres M, Horaitis O, Vanevski M, Cotton RGH (2002) Time for a unified system of mutation description and reporting: a review of locus-specific mutation databases. Genome Research 12 (5):680

10. Kohonen-Corish MR, Al-Aama JY, Auerbach AD, Axton M, Barash CI, Bernstein I, Beroud C, Burn J, Cunningham F, Cutting GR, den Dunnen JT, Greenblatt MS, Kaput J, Katz M, Lindblom A, Macrae F, Maglott D, Moslein G, Povey S, Ramesar R, Richards S, Seminara D, Sobrido MJ, Tavtigian S, Taylor G, Vihinen M, Winship I, Cotton RG (2010) How to catch all those mutations--the report of the third Human Variome Project Meeting, UNESCO Paris, May 2010. Hum Mutat 31 (12):1374-1381. doi:10.1002/humu.21379

11. Patrinos GP, Al Aama J, Al Aqeel A, Al-Mulla F, Borg J, Devereux A, Felice AE, Macrae F, Marafie MJ, Petersen MB, Qi M, Ramesar RS, Zlotogora J, Cotton RG (2011) Recommendations for genetic variation data capture in developing countries to ensure a comprehensive worldwide data collection. Hum Mutat 32 (1):2-9. doi:10.1002/humu.21397

12. Lopes P, Dalgleish R, Oliveira JL (2011) WAVe: Web Analysis of the Variome. Human Mutation 32. doi:10.1002/humu.21499

13. Hamosh A, Scott AF, Amberger JS, Bocchini CA, McKusick VA (2005) Online Mendelian Inheritance in Man (OMIM), a knowledgebase of human genes and genetic disorders. Nucleic acids research 33 (suppl 1):D514

14. Bruford EA, Lush MJ, Wright MW, Sneddon TP, Povey S, Birney E (2008) The HGNC Database in 2008: a resource for the human genome. Nucleic acids research 36 (suppl 1):D445

15. Riikonen P, Vihinen M (1999) MUTbase: maintenance and analysis of distributed mutation databases. Bioinformatics 15 (10):852-859. doi:10.1093/bioinformatics/15.10.852

16. Christophe Béroud, Gwenaîlle Collod-Béroud, Catherine Boileau, Thierry Soussi, Claudine Junien (2000) UMD (Universal Mutation Database): A generic software to build and analyze locus-specific databases. Human Mutation 15 (1):86-94

17. Ivo F.A.C. Fokkema, Johan T. den Dunnen, Peter E.M. Taschner (2005) LOVD: Easy creation of a locus-specific sequence variation database using an ldquoLSDB-in-a-boxrdquo approach. Human Mutation 26 (2):63-68 